Kommentar zum „Haus des Wissens"

\title{
Ärztliche Fachgesellschaften - die Garanten ärztlichen Wissensmanagements
}

Die exponenzielle Zunahme des medizinischen Wissens ist Charakteristikum und Herausforderung für das Gesundheitswesen der Gegenwart. Herausforderung deshalb, weil Wissenszuwachs und Wissensanwendung in der Patientenversorgung nicht mehr Hand in Hand gehen: Der Arzt schuldet seinem Patienten eine Behandlung nach dem aktuellen Stand der medizinischen Wissenschaft. Dabei kann die Fülle an täglich neu publizierten Studien und anderen versorgungsrelevanten Informationen nur noch durch Unterstützung von Experten erfasst und auf Zuverlässigkeit sowie Praktikabilität geprüft werden.

Ärzte sind nicht mehr in der Lage, die für sie relevanten Innovationen zeitgerecht aus der Vielfalt der verschiedenen Medien herauszufiltern, zu bewerten und in den Versorgungsalltag der Patientenversorgung zu integrieren. Denn häufig verfolgen diejenigen, die neues Wis-

sen hervorbringen und bereitstellen, handfeste wirtschaftliche Interessen. Das heißt: Auf viele Studi-

"Ärztliche Fachgesellschaften sollen den Transfer zuverlässigen Wissens an ihre Mitglieder garantieren."

litätsbewertung neuen Wissens ausgewiesen sind, können hier unterstützend hilfreich sein, wie etwa die Cochrane Collaboration und in Deutschland das Institut für Qualität und Wirtschaftlichkeit im Gesundheitswesen (IQWiG) und das Ärztliche Zentrum für Qualität in der Medizin (äzq). Die wissenschaftlichen medizinischen Fachgesellschaften sollten „Wissensmanagement" noch mehr als bisher zu einer ihrer Kernaufgaben machen. Ärztliche Fachgesellschaften können ihre Mitglieder dazu befähigen,,,evidenzbasierte Entscheidungen" zu fällen. Dabei verstehe ich "evidenzbasiert" als "wissenschaftlich fundierte und vertrauenswürdige Erkenntnis", die nicht von ökonomischen und nicht patientenorientierten Interessen Dritter beeinflusst wird.

Voraussetzung könnte ein Informationsdienst einer Fachgesellschaft, zum Beispiel der DGU, sein. Ein Informationsdienst, der aktuell ist, der die Informationsbedürfnisse der Mitglieder berücksichtigt, der die Chancen und en und ihre Ergebnisse ist kein Verlass. Mit dem entsprechenden Studiendesign, der Wahl der Endpunkte oder der Vergleichstherapie lassen sich Ergebnisse völlig unterschiedlich darstellen. Studien, die von der Industrie bezahlt wurden, bewerteten häufiger einen Effekt als signifikant und klinisch relevant [Booth CM et al. Evolution of the Randomized Clinical Trial in Oncology Over Three Decades. Journal of Clinical Oncology 2008; 26: 5458-64].

In dem aktuellen Informationsdickicht hochwertiges von nutzlosem Wissen zu unterscheiden, wird für jeden Arzt täglich schwerer. Und doch muss er entscheiden, auf welches Wissen er sein Handeln gründet, nach welchen Erkenntnissen er seine Patienten behandeln soll.

Hier müssen wissenschaftliche Fachgesellschaften helfen. Ärztliche Fachgesellschaften sollen den Transfer zuverlässigen Wissens an ihre Mitglieder garantieren. Institutionen, die in der Recherche und Qua- damit von den wissenschaftlichen Fachgesellschaften) noch intensiver als bisher als ernstzunehmende Ressource behandelt werden [nach Antes, faz.net 20.6.2011, „, Für Patienten forschen, nicht für die Bilanzen"]. Der Qualität der Versorgung, den Patienten, dem Renommee der Fachgesellschaften und der ärztlichen Professionalität würde es gut tun.

Die DGU ist hier exzellent aufgestellt: Die seit Jahren bestehende Kooperation mit dem äzq hat die hochwertige und allseits beachtete Leitlinie „Interdisziplinäre Leitlinie der Qualität S3 zur Früherkennung, Diagnose und Therapie der verschiedenen Stadien des Prostatakarzinoms hervorgebracht (www. krebsgesellschaft.de/download/s3-leitlinieprostatakarzinom.pdf). Davon profitieren nicht nur die urologisch tätigen Ärzte, sondern auch ihre Patienten. Diese Kooperation könnte im Sinne eines umfassenden Wissensmanagements der DGU weiterentwickelt werden.

\section{Prof. Dr. Günter Ollenschläger}

Leiter des äzq (Ärztliches Zentrum für

Qualität in der Medizin)

Gemeinsames Institut von

Bundesärztekammer und

Kassenärztlicher Bundesvereinigung, Berlin Internet: www.aezq.de

E-Mail:mail@azq.de tisch diskutiert. Grundlage eines solchen Wissensmanagement-Programms wissenschaftlich medizinischer Fachgesellschaften wären:

_ ein Programm der Fachgesellschaft zur Datenakquise im Rahmen der Versorgungsforschung (Wissensgenerierung);

_ die Sichtung und Beurteilung von Forschungsergebnissen aus Versorgungsrealität und Studien durch einschlägige methodisch qualifizierte Institutionen (Wissensaufbereitung);

_ die Kommentierung und Verbreitung der so zusammengetragenen Erkenntnisse (Wissenstransfer).

„Sauberes", also zuverlässiges Wissen und dessen intelligente Nutzung in der Patientenversorgung als "einziger unbegrenzter und erneuerbarer medizinischer Rohstoff " sollte im deutschen Gesundheitssystem (und 